Z. Wahrscheinlichkeitstheorie verw. Gebiete 64. 275-301 (1983)
Zeitschrift für

Wahrscheinlichkeitstheorie und verwandte Gebiete

(C) Springer-Verlag 1983

\title{
Fixed Points of the Smoothing Transformation
}

\author{
Richard Durrett and Thomas M. Liggett* \\ Mathematics Department, University of California, Los Angeles, California 90024, USA
}

\begin{abstract}
Summary. Let $W_{1}, \ldots, W_{N}$ be $N$ nonnegative random variables and let $\mathfrak{M}$ be the class of all probability measures on $[0, \infty)$. Define a transformation $T$ on $\mathfrak{M}$ by letting $T \mu$ be the distribution of $W_{1} X_{1}+\ldots+W_{N} X_{N}$, where the $X_{i}$ are independent random variables with distribution $\mu$, which are independent of $W_{1}, \ldots, W_{N}$ as well. In earlier work, first Kahane and Peyriere, and then Holley and Liggett, obtained necessary and sufficient conditions for $T$ to have a nontrivial fixed point of finite mean in the special cases that the $W_{i}$ are independent and identically distributed, or are fixed multiples of one random variable. In this paper we study the transformation in general. Assuming only that for some $\gamma>1, E W_{i}^{\gamma}<\infty$ for all $i$, we determine exactly when $T$ has a nontrivial fixed point (of finite or infinite mean). When it does, we find all fixed points and prove a convergence result. In particular, it turns out that in the previously considered cases, $T$ always has a nontrivial fixed point. Our results were motivated by a number of open problems in infinite particle systems. The basic question is: in those cases in which an infinite particle system has no invariant measures of finite mean, does it have invariant measures of infinite mean? Our results suggest possible answers to this question for the generalized potlatch and smoothing processes studied by Holley and Liggett.
\end{abstract}

\section{Introduction}

A number of authors have studied interacting particle systems in which the state of each site $x \in Z^{d}$ is described by a nonnegative real number $([5,11,14]$, for example), or more generally the state of the system is a random measure on

\footnotetext{
* The research of both authors was supported in part by NSF Grant MCS 80-02732. The first author is an Alfred P. Sloan fellow
} 
$R^{d}([1,2,7,8,10,15])$. In most cases it has only been possible to find sufficient conditions for the existence and for the nonexistence of invariant measures of finite mean. Once there is one invariant measure of finite mean there is normally at least a one parameter family of them indexed by the mean. A familiar example is "independent motions" where the invariant measures are Poisson processes with mean measures $\lambda d x, 0 \leqq \lambda<\infty$.

As in the last example, the existence of a one parameter family of invariant measures with finite mean usually makes it possible to rule out the existence of nontrivial invariant measures with infinite mean. The idea is to show that any extremal invariant with infinite mean must be stochastically larger than all the members of the known one parameter family and hence must be the pointmass on the configuration which is identically infinity. When there is no invariant measure of finite mean (e.g. branching random walks in $d \leqq 2$ ), it is natural to ask whether the process has invariant measures of infinite mean. However, the ideas used in the finite mean case simply do not shed much light on this. In most cases, it has been difficult even to formulate a reasonable conjecture concerning this question. Examples of situations in which this question has been raised either explicitly or implicitly are: (i) open problem $b$ of Sect. 8 of Holley and Liggett (1981) for generalized potlatch and smoothing processes, (ii) conjecture 1.6 of Liggett (1978) for independent particle systems, (iii) Theorem 1.2 of Kallenberg (1977) for critical cluster fields, and (iv) Theorem 3.1 of Dawson (1977) for measure diffusion processes. (In the latter two cases, the question is implicit in the finite intensity assumptions in the theorems. In Dawson's theorem, this assumption appears in the proof rather than in the statement of the theorem.)

This paper is an attempt to shed some light on the above question by considering a simplified version of the generalized smoothing process of Holley and Liggett (1981), which we will call the smoothing transformation. In order to define the smoothing transformation fix $N$ nonnegative random variables $W_{1}, \ldots, W_{N}$ with $P\left(W_{i}>0\right)>0$, but which otherwise have an arbitrary joint distribution, and let $\mathfrak{M}$ be the class of all probability measures on $[0, \infty)$. The smoothing transformation $T$ on $\mathfrak{M}$ is defined by letting $T \mu$ be the distribution of $W_{1} X_{1}+\ldots+W_{N} X_{N}$, where $X_{1}, \ldots, X_{N}$ are independent random variables with distribution $\mu$, and are independent of $\left(W_{1}, \ldots, W_{N}\right)$. Of course, $T$ can be regarded as a (nonlinear) transformation on the class $\mathfrak{L}$ of Laplace transforms $\varphi$ of elements of $\mathfrak{M}$. With this interpretation, $T$ takes the form

$$
(T \varphi)(\theta)=E \prod_{i=1}^{N} \varphi\left(\theta W_{i}\right) .
$$

Kahane and Peyriere (1976) gave necessary and sufficient conditions for $T$ to have a fixed point of finite mean in the case that $W_{1}, \ldots, W_{N}$ are independent and identically distributed. Their work was motivated by questions raised by Mandelbrot relating to a model for turbulence. Holley and Liggett (1981) did the same in the case that $W_{1}, \ldots, W_{N}$ are constant multiples of a fixed random variable. In their case, the problem was motivated by the connection between the smoothing transformation $T$ and the generalized smoothing process. 
In this paper we will study the transformation in general assuming only that for some $\gamma>1, E W_{i}^{\gamma}<\infty$ for all $i$ and will determine when $T$ has fixed points of finite or infinite mean. To state our results we will need a number of definitions. Let $\mathfrak{F}$ be the set of all nontrivial fixed points of $T$ :

$$
\mathfrak{F}=\left\{\mu \in \mathfrak{M}: T \mu=\mu \text { and } \mu \neq \delta_{0}\right\} .
$$

The condition for the existence of a nontrivial fixed point for $T$ is given in terms of the function

$$
v(\alpha)=\log \left(\sum_{i=1}^{N} E\left(W_{i}^{\alpha} ; W_{i}>0\right)\right)
$$

which is defined for all $\alpha \geqq 0$ and is finite for $0 \leqq \alpha \leqq \gamma$.

Theorem 1. $\mathfrak{F} \neq \emptyset$ if and only if for some $\alpha \in(0,1], v(\alpha)=0$ and $v^{\prime}(\alpha) \leqq 0$.

Although the statement of this result is a simple dichotomy, its proof is not a two part affair - it is a combination of five propositions: (2.7), (2.12), (3.1), (3.2), and (3.5). Perhaps the best way of indicating the elements of the proof and how they fit together is to outline the argument for the example which led us to the theorem stated above.

Suppose $N=2$ and $W_{1}=W_{2}$ with distribution given by

$$
P\left(W_{i}=A\right)=p, \quad P\left(W_{i}=A^{-1}\right)=1-p
$$

for some $A>1$ and $p \in(0,1)$. In this example

$$
v(\alpha)=\log \left(2\left[p A^{\alpha}+(1-p) A^{-\alpha}\right]\right)
$$

so by Theorem $1, \mathfrak{F} \neq \emptyset$ if and only if for some $\alpha \in(0,1]$,

$$
u(\alpha) \equiv 2\left[p A^{\alpha}+(1-p) A^{-\alpha}\right]=1
$$

and

$$
v^{\prime}(\alpha)=\frac{u^{\prime}(\alpha)}{u(\alpha)}=\frac{2\left(p A^{\alpha}-(1-p) A^{-\alpha}\right) \ln A}{2\left(p A^{\alpha}+(1-p) A^{-\alpha}\right)} \leqq 0
$$

This conclusion was arrived at by considering four cases.

Case 1. $v(1)=0$. In this case

$$
E\left(W_{1}+W_{2}\right)=2\left[p A+(1-p) A^{-1}\right]=u(1)=1
$$

so a result of Holley and Liggett (1978) (see Theorem 7.1 on p. 190) can be applied to conclude that there are fixed points of finite mean if and only if

$$
E\left(2 W_{i} \log \left(2 W_{i}\right)\right)<-2\left(\frac{1}{2} \log \frac{1}{2}\right)=\log 2
$$

(the analogous transformation in their setting is $T X=W\left(X_{1}+X_{2}\right) / 2$ ). If we observe that $E\left(2 W_{i}\right)=1$ we can write the condition above as $2 E\left(W_{i} \log W_{i}\right)<0$ or in view of Theorem 1 and the fact that 


$$
v^{\prime}(1)=u^{\prime}(1)=2\left(p A \ln A+(1-p) A^{-1} \ln A^{-1}\right)
$$

we can write the condition as $v^{\prime}(1)<0$.

In the last simplification we have relied on knowing the solution of the problem. Even before solving the problem however, it is easy to see that the transition between existence and nonexistence of fixed points of finite mean occurs at a special point.

In order for the equality $v(1)=0$ above to hold, we must have $2 p A^{2}-A$ $+2(1-p)=0$, i.e.

$$
A=\frac{1 \pm \sqrt{1-16 p(1-p)}}{4 p}
$$

In order for this to be real and larger than one, we must have $p \leqq p_{c}=[$ the smaller root of $16 p(1-p)]=(2-\sqrt{3}) / 4 \approx 0.067$. When $p<p_{c}$ there are two possible values of $A$. Checking the value of $v^{\prime}(\alpha)$ one finds that the smaller value of $A$ always has $v^{\prime}(\alpha)<0$ and the larger value always has $v^{\prime}(\alpha)>0$ so the cutoff corresponds to the point on the curve $2\left(p A+(1-p) A^{-1}\right)=1$ which is above $p_{c}$. This curve is sketched in Fig. 1.

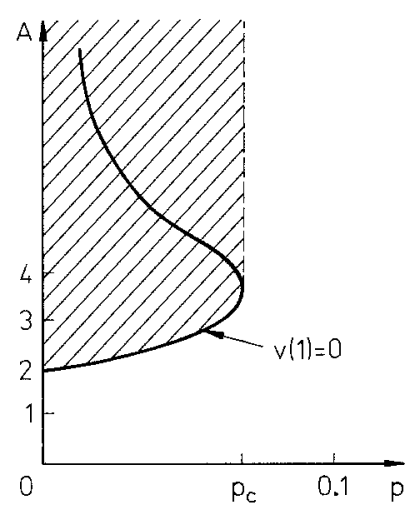

Fig. 1

Case II. (3.1) Suppose $v(\alpha)=0$ and $v^{\prime}(\alpha)<0$ for some $\alpha \in(0,1)$. Then $\mathfrak{F} \neq \emptyset$ (this covers the shaded region in Fig. 1). Define $\tilde{W}_{i}=W_{i}^{\alpha}$ and let $\tilde{v}, \tilde{T}$, and $\tilde{\mathbb{F}}$ be what results when we replace $W_{i}$ by $\tilde{W}_{i}$. It is easy to check that $\tilde{v}(1)=0$ and $\tilde{v}^{\prime}(1)<0$ so by Case $I$, there is a $\psi \in \tilde{\widetilde{F}}$ with finite mean. Let $\varphi(\theta)=\psi\left(\theta^{\alpha}\right)$. If we let $X(t)$ be the one sided stable process with index $\alpha$ and let $\tau$ be an independent random variable with Laplace transform $\psi$ then $\varphi(\theta)=E \exp (-\theta X(\tau))$ so $\varphi$ is a Laplace transform. It is easy to check that $T \varphi=\varphi$.

Case III. (3.5) Suppose that $v(\alpha)=0$ and $v^{\prime}(\alpha)=0$ for some $\alpha \in(0,1]$. Then $\mathbb{F} \neq \emptyset$ (this covers the dotted line at the border of the shaded region in Fig. 1). Since $v$ is strictly convex, $v(\beta)>0$ and $v^{\prime}(\beta)<0$ for all $\beta<\alpha$. Let $W_{i, \beta}=W_{i} \exp (-v(\beta) / \beta)$ and $v_{\beta}, T_{\beta}$ and $\mathfrak{F}_{\beta}$ be what results when we replace $W_{i}$ by $W_{i, \beta}$. Then $v_{\beta}(\beta)=0$ 
and $v_{\beta}^{\prime}(\beta)<0$ so $T_{\beta}$ has a nontrivial fixed point $\psi_{\beta}$ by Case II. It turns out (see (3.2)) that $\psi_{\beta}(\infty)=t_{0}$ is independent of $\beta$ so by scaling we can choose the fixed point $\psi_{\beta}$ so that $\psi_{\beta}(1)=\left(t_{0}+1\right) / 2$ and hence we can let $\beta \uparrow \alpha$ to construct a nontrivial fixed point for $T$.

Case IV. (2.12a) If $\mathfrak{F} \neq \emptyset$ then there is an $\alpha \in(0,1]$ so that $v(\alpha)=0$. (This shows that Cases I-III give us all the cases in which fixed points exist.) Let $D_{\alpha}(x)$ $=e^{\alpha x}\left(1-\varphi\left(e^{-x}\right)\right)$. If we let $Y_{\alpha}$ be a random variable with distribution given by

and let

$$
E f\left(Y_{\alpha}\right)=e^{-v(\alpha)} \sum_{i=1}^{N} E\left[f\left(-\log W_{i}\right) W_{i}^{\alpha} ; W_{i}>0\right]
$$

$$
G_{\alpha}(x)=e^{\alpha x} E\left(\prod_{i=1}^{N} \varphi\left(e^{-x} W_{i}\right)-1+\sum_{i=1}^{N}\left(1-\varphi\left(e^{-x} W_{i}\right)\right)\right)
$$

then a little calculation (see (2.3)) shows that

$$
D_{\alpha}(x)=e^{v(\alpha)} E D_{\alpha}\left(x+Y_{\alpha}\right)-G_{\alpha}(x) .
$$

Replacing $x$ by $x+y$ in the last expression, dividing both sides by $D_{a}(x)$, and setting $h_{x}(y)=D_{\alpha}(x+y) / D_{\alpha}(x)$ gives

$$
h_{x}(y)=e^{v(\alpha)} E h_{x}\left(y+Y_{\alpha}\right)-\frac{G_{\alpha}(x)}{D_{\alpha}(x)} .
$$

If the second term on the right hand side were not there then we would know all the solutions of this equation. To get rid of it we let $x \rightarrow \infty$ and show

(i) $h_{x}(\cdot), x \in \mathbb{R}$, are uniformly bounded and equicontinuous on compact subsets, so subsequential limits exist,

(ii) if $h$ is a subsequential limit then

and consequently

$$
h(y)=e^{v(\alpha)} E h\left(y+Y_{\alpha}\right)
$$

(iii) $h$ must be $c_{1} e^{\left(\alpha-\beta_{1}\right) x}+c_{2} e^{\left(\alpha-\beta_{2}\right) x}$ where $\beta_{1}$ and $\beta_{2}$ are the (at most two) $\beta$ 's which have $v(\beta)=0$. That these $\beta$ 's lie in $(0,1]$ follows from the monotonicity and convexity of $\varphi$.

The last statement is possible only if $v(\beta)=0$ has at least one root in $(0,1]$ so this completes Case IV. To close the gap between this and the existence result we observe that if $\alpha$ is the smaller root then the convexity of $v$ implies that $v^{\prime}(\alpha) \leqq 0$.

Having found a necessary and sufficient condition for the existence of fixed points the next logical step is to describe the set of all fixed points. As we mentioned before, when there is a fixed point of finite mean there is typically exactly a one parameter family of them, so given the method of constructing fixed points in Cases II and III it seems natural to conjecture that is also the case in general. This turns out to be true if one considers "typically" to mean "under suitable irreducibility assumptions."

We will say that the problem is of lattice type if there is an $s>0$ so that 
with probability one each $\log W_{i}$ is an integer multiple of $s$. We will always take $s$ to be the largest possible such number and will refer to it as the span. If the problem is nonlattice we will set $s=0$. The set of all fixed points is parametrized by a class $\mathfrak{P}_{\alpha, s}$ which we will now define. If $s>0$ and $\alpha \in(0,1)$, let $\mathfrak{P}_{\alpha, s}$ be the collection of all strictly positive infinitely differentiable functions $p$ on $R^{1}$ which satisfy

(a) $p(x+s)=p(x)$ for all $x \in R^{1}$, and

(b) $(-1)^{k} \frac{d^{k}}{d \theta^{k}}\left[\theta^{\alpha} p(-\log \theta)\right] \leqq 0$

for all $k=1,2, \ldots$ and all $\theta \in(0, \infty)$. As will be seen in Sect. $5, \mathfrak{P}_{\alpha, s}$ is rather large for these choices of $\alpha$ and $s$. If $s=0$ and $\alpha \in(0,1]$ or if $\alpha=1$ and $s>0$, let $\mathfrak{P}_{\alpha, s}$ be the set of positive constant functions on $R^{1}$. (It is interesting to note that periodicities play a role if $\alpha<1$, but not if $\alpha=1$.)

Note that $v \equiv 0$ if and only if $P\left(W_{i}=1\right)+P\left(W_{i}=0\right)=1$ for each $i$ and $\sum_{i=1}^{N} P\left(W_{i}=1\right)=1$, and in this case $\mathfrak{F}=\mathfrak{M} \backslash\left\{\delta_{0}\right\}$. Thus in the next result we will assume that $v$ is not identically zero, in which case there is at most one $\alpha \in(0,1]$ at which $v(\alpha)=0$ and $v^{\prime}(\alpha) \leqq 0$. When $\mathfrak{F} \neq \emptyset$, Theorem 1 guarantees that there is exactly one such $\alpha$.

Theorem 2. Suppose that $v$ is not identically zero and that $\mathfrak{F} \neq \emptyset$. Let $\alpha$ be the unique point in $(0,1]$ for which $v(\alpha)=0$ and $v^{\prime}(\alpha) \leqq 0$.

(a) Then there is a natural one-to-one correspondence between $\varphi \in \mathbb{F}$ and $p \in \mathfrak{P}_{\alpha, s}$ which is given by

and

$$
\lim _{\theta \downarrow 0} \frac{1-\varphi(\theta)}{\theta^{\alpha} p(-\log \theta)}=1 \quad \text { if } \quad v^{\prime}(\alpha)<0
$$

$$
\lim _{\theta \downarrow 0} \frac{1-\varphi(\theta)}{\theta^{\alpha} p(-\log \theta)|\log \theta|}=1 \quad \text { if } v^{\prime}(\alpha)=0 \text {. }
$$

(b) Suppose $\varphi \in \mathfrak{F}$ and $\psi \in \mathfrak{Q}$. If

then $\lim _{n \rightarrow \infty} T^{n} \psi=\varphi$.

$$
\lim _{\theta \downarrow 0} \frac{1-\varphi(\theta)}{1-\psi(\theta)}=1,
$$

Part (a) says that the fixed points can be identified by the behavior of their Laplace transforms at 0 . When $\alpha=1$ and $v^{\prime}(\alpha)<0$ this just says that the fixed points are parametrized by their means. Part (b) is our convergence theorem. It says that if the behavior of $\psi$ matches that of $\varphi$ at 0 then $T^{n} \psi \rightarrow \varphi$ as $n \rightarrow \infty$. When $\alpha=1$ and $v^{\prime}(\alpha)<0$ this just says that the two random variables have the same mean. In other cases the condition is more restrictive, but this situation is analogous to that for sums of independent random variables: when the variance is finite, $\left(S_{n}-n m\right) / n^{1 / 2}$ converges to a normal, but when we deal with convergence to stable laws we require special assumptions on the tail of the 
distribution = information about the behavior of the characteristic function at 0 .

Our last result concerns the size of the tails of the distributions of the fixed points in the finite mean case.

Theorem 3. Suppose $v(1)=0$ and $v^{\prime}(1)<0$. Then for any $\mu \in \mathfrak{F}$ and $\beta>1$

$$
\int_{0}^{\infty} x^{\beta} d \mu<\infty \quad \text { if and only if } v(\beta)<0 .
$$

The proof of this is a straightforward generalization of the proof of the corresponding result of Kahane and Peyriere (1976). To see what it says in a concrete case let $W_{i}=e^{Z_{i}}$ where $Z_{i}$ has the normal distribution with mean $m$ and variance $\sigma^{2}>0$. Then $\mathfrak{F} \neq \emptyset$ if and only if

and

$$
m \leqq-\sigma \sqrt{2 \log N} \quad \text { if } \sigma>\sqrt{2 \log N}
$$

$$
m \leqq-\log N-\frac{\sigma^{2}}{2} \quad \text { if } \sigma \leqq \sqrt{2 \log N} .
$$

Elements of $\mathfrak{F}$ have a finite moment of order $\beta \geqq 1$ if and only if $m=-\log N$ $-\frac{\sigma^{2}}{2}$ and $\frac{\beta \sigma^{2}}{2}<\log N$.

It is also interesting to apply this result to the first example considered above. If we let $p<p_{c}$ and $A=(1-\sqrt{1-16 p(1-p)}) / 4 p$ so $v(1)=0$, then as $p \rightarrow 0$ the number of moments which are finite increases to $\infty$. Reversing this and recalling how the fixed points were constructed in Case II we see that as we move up the curve the number of moments which are finite decreases to 0 .

In view of the results of Holley and Liggett (1981), our theorems suggest the following conjecture about the behavior of the generalized smoothing process: the existence or nonexistence of stationary measures should correspond to the transience of recurrence of the underlying symmetrized random walk, while the effect of the spread of $W$ should be only to determine the size of the tails of the stationary measure when it exists. If this were the case, the behavior of the generalized smoothing and potlatch processes would be consistent with the behavior of critical cluster fields (see Kallenberg (1977) for example). Resolving these questions even in special cases seems to be quite difficult.

Perhaps it should be mentioned at this point that the connection between the generalized smoothing process and the smoothing transformation is stronger than mere analogy. To see this connection, consider the generalized smoothing process $\eta_{t}$ corresponding to the random variable $W$ and the simple random walk transition probabilities $p(x, y)=\frac{1}{2 d}$ for $|x-y|=1$ on $Z^{d}$. Suppose the translation invariant initial distribution is a product measure, or more generally, has positive correlations in the sense of Harris (1977). Let $\varphi_{t}(\theta)$ be the Laplace transform of $\eta_{t}(0)$. By Theorem 1.1 of Harris (1977), the distribution of the process at time $t$ has positive correlations as well, so 


$$
\begin{aligned}
\frac{d}{d t} \varphi_{t}(\theta) & =E \exp \left[-\theta \frac{W}{2 d} \sum_{|x|=1} \eta_{t}(x)\right]-\varphi_{t}(\theta) \\
& \geqq \prod_{|x|=1} E \exp \left[-\theta \frac{W}{2 d} \eta_{t}(x)\right]-\varphi_{t}(\theta) \\
& =\left\{E \exp \left[-\theta \frac{W}{2 d} \eta_{t}(0)\right]\right\}^{2 d}-\varphi_{t}(\theta) \\
& =T \varphi_{t}(0)-\varphi_{t}(\theta),
\end{aligned}
$$

where $T$ is the smoothing transformation corresponding to $W_{i}=\frac{W}{2 d}$ for $1 \leqq i \leqq 2 d$. Therefore

$$
\varphi_{t}(\theta) \geqq e^{-t} \sum_{n=0}^{\infty} \frac{t^{n}}{n !} T^{n} \varphi_{0}(\theta),
$$

so that $\lim _{n \rightarrow \infty} T^{n} \varphi_{0}(\theta)=1$ for all $\theta$ implies that $\lim _{n \rightarrow \infty} \varphi_{t}(\theta)$ for all $\theta$.

The proofs of our results are organized according to the tools used. In Sect. 2 the arguments use random walk ideas (potential theory and renewal theory); Section 3 concerns the stable law transformation; Section 4 deals with an associated branching random walk. Section 5 contains the parts of the proofs which do not fit naturally into the earlier sections. Theorem 1 is obtained by combining Theorems $2.7,2.12,3.1,3.2$, and 3.5. Theorem $2 \mathrm{a}$ is obtained from Theorems 2.18, 4.2, and 5.1. Theorems $2 b$ and 3 are just Theorems 4.2 and 5.3 , respectively.

\section{The Associated Random Walk}

Throughout this section, $\alpha$ will always be a point in $(0,1]$. Let $Y_{\alpha}$ be a random variable with distribution determined by

$$
E f\left(Y_{\alpha}\right)=e^{-v(\alpha)} \sum_{i=1}^{N} E\left[f\left(-\log W_{i}\right) W_{i}^{\alpha}, W_{i}>0\right]
$$

for nonnegative continuous functions $f$ on $R^{1}$. This is possible since the right hand side of (2.1) is a positive linear functional with unit norm. The random walk to be used in this section is the one whose increments have the distribution of $Y_{\alpha}$. Initially, the arguments are similar to those used in Holley and Liggett (1981).

Given $\varphi \in \mathscr{L}$ with $\varphi \neq 1$, define $D_{\alpha}(x)$ and $G_{\alpha}(x)$ by

$$
\begin{aligned}
& D_{\alpha}(x)=e^{\alpha x}\left[1-\varphi\left(e^{-x}\right)\right], \quad \text { and } \\
& G_{\alpha}(x)=e^{\alpha x} E\left\{\prod_{i=1}^{N} \varphi\left(e^{-x} W_{i}\right)-1+\sum_{i=1}^{N}\left[1-\varphi\left(e^{-x} W_{i}\right)\right]\right\} .
\end{aligned}
$$

Put $\tilde{\varphi}=T \varphi$, and define $\tilde{D}_{\alpha}$ and $\tilde{G}_{\alpha}$ analogously in terms of $\tilde{\varphi}$.

(2.3) Lemma. $\tilde{D}_{\alpha}(x)=e^{v(\alpha)} E D_{\alpha}\left(x+Y_{\alpha}\right)-G_{\alpha}(x)$.

Proof. Using the appropriate definitions, one checks that 


$$
\begin{aligned}
D_{\alpha}(x) & =e^{\alpha x}\left[1-\tilde{\varphi}\left(e^{-x}\right)\right] \\
& =e^{\alpha x} E\left[1-\prod_{i=1}^{N} \varphi\left(e^{-x} W_{i}\right)\right] \\
& =e^{\alpha x} E \sum_{i=1}^{N}\left[1-\varphi\left(e^{-x} W_{i}\right)\right]-G_{\alpha}(x) \\
& =\sum_{i=1}^{N} E\left[W_{i}^{\alpha} D_{\alpha}\left(x-\log W_{i}\right), W_{i}>0\right]-G_{\alpha}(x) \\
& =e^{v(\alpha)} E D_{\alpha}\left(x+Y_{\alpha}\right)-G_{\alpha}(x) .
\end{aligned}
$$

(2.4) Lemma. (a) $G_{x}(x) \geqq 0$.

(b) $e^{-\alpha x} G_{x}(x)$ is a decreasing function of $x$.

(c) If $\tilde{\varphi} \geqq \varphi$, then $\tilde{G}_{\alpha}(x) \leqq G_{\alpha}(x)$.

Proof. All three statements follow from the following fact: if $0 \leqq u_{i} \leqq v_{i} \leqq 1$, then

$$
\prod_{i=1}^{N} u_{i}-1+\sum_{i=1}^{N}\left(1-u_{i}\right) \geqq \prod_{i=1}^{N} v_{i}-1+\sum_{i=1}^{N}\left(1-v_{i}\right)
$$

To verify this fact, note that

$$
\frac{\partial}{\partial x_{j}}\left[\prod_{i=1}^{N} x_{i}-1+\sum_{i=1}^{N}\left(1-x_{i}\right)\right]=\prod_{i \neq j} x_{i}-1 \leqq 0 .
$$

(2.6) Lemma. (a) $G_{\alpha}(x) \leqq e^{\alpha x} \operatorname{EF}\left(Z D_{\alpha}(x) e^{-\alpha x}\right)$, where $Z=\sum_{i=1}^{N} \max \left(W_{i}, 1\right)$ and

$$
F(u)= \begin{cases}e^{-u}-1+u & \text { if } u \leqq N \\ e^{-N}-1+N & \text { if } u \geqq N\end{cases}
$$

(b) $\lim _{x \rightarrow \infty} \frac{G_{\alpha}(x)}{D_{\alpha}(x)}=0$.

Proof. For part (a), use the inequality $u \leqq e^{-(1-u)}$ to obtain

$$
\begin{aligned}
G_{\alpha}(x) & \leqq e^{\alpha x} E\left\{e^{-\sum_{i=1}^{N}\left[1-\varphi\left(e^{-x} W_{i}\right)\right]}-1+\sum_{i=1}^{N}\left[1-\varphi\left(e^{-x} W_{i}\right)\right]\right\} \\
& \leqq e^{\alpha x} E F\left[\sum_{i=1}^{N}\left[1-\varphi\left(e^{-x} W_{i}\right)\right]\right]
\end{aligned}
$$

since $\sum_{i=1}^{N}\left[1-\varphi\left(e^{-x} W_{i}\right)\right] \leqq N$. Since $\varphi \in \mathfrak{Q}, \frac{1-\varphi(u)}{u}$ is decreasing and $1-\varphi(u)$ is increasing in $u$. Therefore

$$
1-\varphi\left(e^{-x} W_{i}\right) \leqq \max \left(W_{i}, 1\right)\left[1-\varphi\left(e^{-x}\right)\right] .
$$

Part (a) now follows from the monotonocity of $F$ on $[0, \infty)$. For part (b), note that since 


$$
\lim _{x \rightarrow \infty} D_{\alpha}(x) e^{-\alpha x}=\lim _{x \rightarrow \infty}\left[1-\varphi\left(e^{-x}\right)\right]=0
$$

it suffices, by changing variables $t=D_{\alpha}(x) e^{-\alpha x}$, to show that

$$
\lim _{t \rightarrow \infty} \frac{E F(Z t)}{t}=0
$$

But this follows from the dominated convergence theorem since $\frac{F(u)}{u}$ is bounded and tends to zero as $u \downarrow 0$, and since $Z$ has a finite first moment.

(2.7) Theorem. Suppose $v(1)=0$ and $v^{\prime}(1)<0$. Then $\mathfrak{F}$ contains elements of finite mean.

Proof. Put $\varphi_{0}(\theta)=e^{-\theta}$ and define $\varphi_{n}(\theta)$ recursively by $\varphi_{n+1}=T \varphi_{n}$. By Jensen's inequality,

$$
\varphi_{1}(\theta)=E \exp \left[-\theta \sum_{i=1}^{N} W_{i}\right] \geqq \exp \left[-\theta E \sum_{i=1}^{N} W_{i}\right]=\varphi_{0}(\theta),
$$

since $v(1)=0$. Therefore

$$
\varphi_{n+1}(\theta) \geqq \varphi_{n}(\theta)
$$

for all $n$. As a consequence,

$$
\varphi(\theta)=\lim _{n \rightarrow \infty} \varphi_{n}(\theta) \geqq \varphi_{0}(\theta)
$$

exists and is a fixed point for $T$. Since $\varphi(\theta) \geqq e^{-\theta}$, the measure in $\mathfrak{M}$ with Laplace transform $\varphi$ has a mean which is at most one. In order to show that $\varphi \in \mathfrak{F}$, it then suffices to show that $\varphi$ is not identically one. In order to do this, let $D_{n}$ and $G_{n}$ be defined as in (2.2) in terms of $\varphi_{n}$, with $\alpha=1$. By (2.8) and Lemma 2.4 (c), $G_{n+1}(x) \leqq G_{n}(x)$ for all $n \geqq 0$ and $x \in R^{1}$. Since $v(1)=0$, Lemma 2.3 gives

$$
\begin{aligned}
D_{n+1}(x) & =E D_{n}\left(x+Y_{1}\right)-G_{n}(x) \\
& \geqq E D_{n}\left(x+Y_{1}\right)-G_{0}(x) .
\end{aligned}
$$

Iterating this, we see that

$$
D_{n}(x) \geqq E D_{0}\left(x+S_{n}\right)-E \sum_{k=0}^{n-1} G_{0}\left(x+S_{k}\right)
$$

where $S_{n}$ is the random walk with $S_{0}=0$ and increments which are distributed like $Y_{1}$. By (2.1),

$$
E Y_{1}=-\sum_{i=1}^{N} E W_{i} \log W_{i}=-v^{\prime}(1)>0
$$

so that $\lim _{n \rightarrow \infty} S_{n}=+\infty$ a.s. Since $D_{0}(x)$ is bounded and $\lim _{x \rightarrow \infty} D_{0}(x)=1$, 


$$
\lim _{n \rightarrow \infty} E D_{0}\left(x+S_{n}\right)=1
$$

Since $D_{0}(x) \leqq 1$ and $F$ is nondecreasing, Lemma 2.6a gives (recall $\alpha=1$ )

$$
\begin{aligned}
\int_{-\infty}^{\infty} G_{0}(x) d x & \leqq E \int_{-\infty}^{\infty} e^{x} F\left(Z e^{-x}\right) d x \\
& =E \int_{0}^{\infty} Z \frac{F(\tau)}{\tau^{2}} d \tau,
\end{aligned}
$$

which is finite since $E Z<\infty, F(\tau)$ is bounded, and $F(\tau) \sim \frac{1}{2} \tau^{2}$ as $\tau \downarrow 0$. By Lemma $2.4 \mathrm{~b}, e^{-x} G_{0}(x)$ is decreasing in $x$. Therefore $G_{0}$ is directly Riemann integrable, so by the renewal theorem,

$$
\lim _{x \rightarrow \infty} E \sum_{k=0}^{\infty} G_{0}\left(x+S_{k}\right)=0
$$

This, together with (2.9) and (2.10) implies that

$$
\lim _{x \rightarrow \infty} \lim _{n \rightarrow \infty} D_{n}(x)=1 .
$$

Therefore, by the definition of $D_{n}(x)$,

$$
\lim _{x \rightarrow \infty} e^{x}\left[1-\varphi\left(e^{-x}\right)\right]=1,
$$

so $\varphi$ is the Laplace transform of a distribution of mean one. This completes the proof of the theorem.

The next theorem involves ideas from the Martin boundary theory for the random walk which corresponds to $Y_{\alpha}$. In preparation for that we need the following lemma.

(2.11) Lemma. Fix $\alpha \in(0,1]$ and assume that the problem is nonlattice. Let $\mathfrak{H}_{\alpha}$ be the set of all functions $g$ on $R^{1}$ which satisfy (i) $g(0)=1$, (ii) $g(y) e^{-\alpha y}$ is decreasing in $y$, (iii) $g(y) e^{(1-x) y}$ is increasing in $y$, and (iv)

$$
g(y)=e^{v(\alpha)} E g\left(y+Y_{\alpha}\right) .
$$

Then $\mathfrak{H}_{\alpha}$ is the set of convex combinations of $g_{\beta}(y)=e^{(\alpha-\beta) y}$ for the (at most two) $\beta$ 's which satisfy $0 \leqq \beta \leqq 1$ and $v(\beta)=0$.

Proof. It is easy to check that $g_{\beta} \in \mathfrak{S}_{\alpha}$ if and only if $0 \leqq \beta \leqq 1$ and $v(\beta)=0$. Therefore the statement of the lemma is immediate if $\mathfrak{S}_{\alpha}=\emptyset$. Assume then that $\mathfrak{S}_{\alpha}$ is not empty. $\mathfrak{H}_{\alpha}$ is a compact convex subset of $C\left(R^{1}\right)$ with the topology of uniform convergence on bounded sets. Hence it is the closed convex hull of its extreme points by the Krein-Milman theorem (see Royden (1968), for example). Suppose that $g$ is an extreme point of $\mathfrak{H}_{\alpha}$ and let

$$
g_{u}(y)=\frac{g(u+y)}{g(u)} .
$$

By property (iv) of the definition of $\mathfrak{S}_{\alpha}$ and the fact that $E g\left(Y_{\alpha}\right)=e^{-v(\alpha)}$, 


$$
g(y)=\frac{E g\left(y+Y_{\alpha}\right)}{E g\left(Y_{\alpha}\right)}=\frac{\int_{-\infty}^{\infty} g_{u}(y) g(u) P\left[Y_{\alpha} \in d u\right]}{\int_{-\infty}^{\infty} g(u) P\left[Y_{\alpha} \in d u\right]} .
$$

Since $g_{u} \in \mathfrak{H}_{\alpha}$ for each $u$ and $g$ is extremal, this implies that $g=g_{u}$ for all $u$ in the support of the distribution of $Y_{\alpha}$. Therefore

$$
g(u+y)=g(u) g(y)
$$

for all $y$ and all $u$ in the support of $Y_{\alpha}$. Since $g$ is continuous and the problem is nonlattice, it follows that $g=g_{\beta}$ for some $\beta$, thus completing the proof of the lemma.

(2.12) Theorem. Suppose that $\varphi \in \mathfrak{F}$. Then (a) there is an $\alpha \in[0,1]$ so that $v(\alpha)$ $=0$, and (b) if $v$ is not identically zero and $\alpha \in(0,1]$ is such that $v(\alpha)=0$ and $v^{\prime}(\alpha) \leqq 0$, then

and

$$
\limsup _{x \rightarrow \infty} \frac{D_{\alpha}(x+y)}{D_{\alpha}(x)} \leqq 1 \quad \text { if } \quad v^{\prime}(\alpha)<0
$$

$$
\lim _{x \rightarrow \infty} \frac{D_{\alpha}(x+y)}{D_{\alpha}(x)}=1 \quad \text { if } \quad v^{\prime}(\alpha)=0
$$

where $y>0$ is any multiple of $s$ if the problem is of lattice type, and is arbitrary otherwise.

Proof. Fix an $\alpha \in(0,1]$, and put

By Lemma 2.3,

$$
h_{x}(y)=\frac{D_{\alpha}(x+y)}{D_{\alpha}(x)}
$$

$$
D_{\alpha}(x)=e^{v(\alpha)} E D_{\alpha}\left(x+Y_{\alpha}\right)-G_{\alpha}(x) .
$$

Evaluating this at $(x+y)$ and dividing by $D_{\alpha}(x)$ gives

$$
h_{x}(y)=e^{v(\alpha)} E h_{x}\left(y+Y_{\alpha}\right)-\frac{G_{\alpha}(x+y)}{D_{\alpha}(x+y)} h_{x}(y) .
$$

Since $\varphi \in \mathfrak{R}, D_{\alpha}(x) e^{-\alpha x}=1-\varphi\left(e^{-x}\right)$ is decreasing in $x$ and $D_{\alpha}(x) e^{(1-\alpha) x}$ is increasing in $x$. Therefore

$$
-(1-\alpha) D_{\alpha}(x) \leqq D_{\alpha}^{\prime}(x) \leqq \alpha D_{\alpha}(x)
$$

so evaluating this at $(x+y)$ and dividing by $D_{\alpha}(x)$ gives

$$
-(1-\alpha) h_{x}(y) \leqq h_{x}^{\prime}(y) \leqq \alpha h_{x}(y)
$$

Since $h_{x}(0)=1$, it follows that

$$
h_{x}(y) \leqq \begin{cases}e^{\alpha y} & \text { for } y>0 \\ e^{-(1-\alpha) y} & \text { for } y<0\end{cases}
$$


which by the previous inequalities gives bounds on $h_{x}^{\prime}(y)$. In particular, the collection $\left\{h_{x}(\cdot), x \in R^{1}\right\}$ is uniformly bounded and equicontinuous on bounded subsets of $R^{1}$, and hence is relatively compact in the topology of uniform convergence on bounded sets. By the definition of $Y_{\alpha}$,

$$
\begin{aligned}
E\left(e^{\alpha Y_{\alpha}}\right) & =e^{v(0)-v(\alpha)}<\infty, \quad \text { and } \\
E\left(e^{-(1-\alpha) Y_{\alpha}}\right) & =e^{v(1)-v(\alpha)}<\infty .
\end{aligned}
$$

Now suppose $x_{n} \rightarrow \infty$ and $h_{x_{n}}(y) \rightarrow h(y)$ uniformly on bounded sets of $R^{1}$. Then by Lemma $2.6 \mathrm{~b}$ and the dominated convergence theorem, we may pass to the limit in (2.13) to obtain

$$
h(y)=e^{v(\alpha)} E h\left(y+Y_{\alpha}\right) .
$$

Assume from now on that the problem is nonlattice. The lattice case is similar. Then $h \in \mathfrak{S}_{\alpha}$, so by Lemma 2.11 , there is a $\beta \in[0,1]$ for which $v(\beta)=0$. This proves part (a) of the theorem. For part (b), suppose now that $v(\alpha)=0$ and $v^{\prime}(\alpha) \leqq 0$. Since we are in the nonlattice case, $v$ is not identically zero. By the convexity of $v$, if $v(\beta)=0$ then $\beta \geqq \alpha$ if $v^{\prime}(\alpha)<0$ and $\beta=\alpha$ if $v^{\prime}(\alpha)=0$. Therefore by Lemma 2.11, $h(y)$ is decreasing in $y$ if $v^{\prime}(\alpha)<0$ and is constant if $v^{\prime}(\alpha)=0$. Since $h(0)=1$, it follows that $h(y) \leqq 1$ if $y>0$ when $v^{\prime}(\alpha)<0$ and $h(y)=1$ when $v^{\prime}(\alpha)=0$. Since this is true for all limit points of $h_{x}(y)$ as $x \rightarrow \infty$, the proof of the theorem is complete.

(2.17) Corollary. Suppose that $\varphi \in \mathfrak{F}, \alpha \in(0,1], v(\alpha)=0, v^{\prime}(\alpha) \leqq 0$, and $v$ is not identically zero. Then $G_{\alpha}(x)$ is directly Riemann integrable on $R^{1}$. (See p. 348 of Feller (1966) for the definition.)

Proof. By Lemma $2.4 \mathrm{~b}, e^{-\alpha x} G_{\alpha}(x)$ is decreasing in $x$. The first step is to use this monotonicity to show that if $G_{\alpha}$ is integrable, then it is directly Riemann integrable. To do this, take $h>0$, and let $\underline{m}_{n}(h)$ and $\bar{m}_{n}(h)$ be the minimum and maximum values of $G_{\alpha}$ on the interval $[(n-1) h, n h]$. Then by the monotonicity of $e^{-\alpha x} G_{\alpha}(x)$,

and

$$
\bar{m}_{n}(h) \leqq e^{\alpha h} G_{\alpha}[(n-1) h]
$$

Therefore

$$
\underline{m}_{n}(h) \geqq e^{-\alpha h} G_{\alpha}[n h] \text {. }
$$

$$
\begin{aligned}
\sum_{n=L}^{M}\left[\bar{m}_{n}(h)-\underline{m}_{n}(h)\right] & \leqq e^{\alpha h} \sum_{n=L-1}^{M-1} G_{\alpha}[n h]-e^{-\alpha h} \sum_{n=L}^{M} G_{\alpha}[n h] \\
& \leqq\left[e^{\alpha h}-e^{-\alpha h}\right] \sum_{n=-\infty}^{\infty} G_{\alpha}[n h]+e^{\alpha h} G_{\alpha}((L-1) h) .
\end{aligned}
$$

Using the monotonicity of $e^{-\alpha} G_{\alpha}(x)$ again,

$$
\int_{(n-1) h}^{n h} G_{\alpha}(x) \geqq h e^{-\alpha h} G_{\alpha}(n h) .
$$

Therefore if $\int_{-\infty}^{\infty} G_{\alpha}(x) d x<\infty$, it follows that $\sum_{n} \bar{m}_{n}(h)<\infty$ and $\sum_{n} \underline{m}(h)<\infty$, and 
that

$$
h \sum_{n=-\infty}^{\infty}\left[\bar{m}_{n}(h)-\underline{m}_{n}(h)\right] \leqq\left(e^{2 \alpha h}-1\right) \int_{-\infty}^{\infty} G_{\alpha}(x) d x .
$$

Since the right side of this inequality tends to zero as $h \downarrow 0, G_{\alpha}$ is directly Riemann integrable. To show that $G_{\alpha}$ is integrable, note that by Lemmas $2.4 \mathrm{a}$ and $2.6 \mathrm{a}$,

$$
0 \leqq G_{\alpha}(x) \leqq e^{\alpha x} E F\left(Z D_{\alpha}(x) e^{-\alpha x}\right)
$$

Since $F$ is bounded,

$$
\int_{-\infty} G_{\alpha}(x) d x<\infty
$$

To deal with integrability at $+\infty$, choose $\beta>0$ so small that $\beta<\alpha / 2$ and $\frac{\alpha}{\alpha-\beta} \leqq \gamma$. Since $D_{\alpha}(x) e^{-\alpha x}$ is decreasing in $x$, Theorem $2.12 \mathrm{~b}$ implies that

$$
\limsup _{x \rightarrow \infty} \frac{1}{x} \log D_{\alpha}(x) \leqq 0 .
$$

Therefore there is an $x_{0}$ so that for $x \geqq x_{0}$

Since $F$ is monotone,

$$
D_{\alpha}(x) \leqq e^{\beta x} .
$$

$$
G_{\alpha}(x) \leqq e^{\alpha x} E F\left(Z e^{(\beta-\alpha) x}\right)
$$

for $x \geqq x_{0}$. By making a change of variable $u=Z e^{(\beta-\alpha) x}$ in the integral and using Fubini's theorem (the integrand is nonnegative),

$$
\begin{aligned}
\int_{x_{0}}^{\infty} G_{\alpha}(x) d x & \leqq \int_{x_{0}}^{\infty} e^{\alpha x} E F\left(Z e^{(\beta-\alpha) x}\right) d x \\
& =E \int_{x_{0}}^{\infty} e^{\alpha x} F\left(Z e^{(\beta-\alpha) x}\right) d x \\
& \leqq E \int_{0}^{\infty} \frac{Z^{\alpha /(\alpha-\beta)}}{\alpha-\beta} \frac{F(u)}{u^{1+(\alpha /(\alpha-\beta))}} d u .
\end{aligned}
$$

The last integral is finite since $E Z^{\alpha /(\alpha-\beta)}<\infty, F$ is bounded, and $F(u) \sim u^{2} / 2$ as $u \downarrow 0$.

The following result is the key to identifying the elements of $\mathfrak{F}$.

(2.18) Theorem. Suppose that $v$ is not identically zero and that $\alpha \in(0,1]$ satisfies $v(\alpha)=0$ and $v^{\prime}(\alpha) \leqq 0$. If $\varphi \in \mathfrak{F}$ then there is a $p \in \mathfrak{P}_{\alpha, s}$ so that
(a) $\lim _{\theta \downarrow 0} \frac{1-\varphi(\theta)}{\theta^{\alpha} p(-\log \theta)}=1$ if $v^{\prime}(\alpha)<0$, and
(b) $\lim _{\theta \downarrow 0} \frac{1-\varphi(\theta)}{\theta^{\alpha}|\log \theta| p(-\log \theta)}=1$ if $v^{\prime}(\alpha)=0$. 
Proof. Let $S_{n}$ be the random walk with $S_{0}=0$ whose increments have distribution $Y_{\alpha}$. Since $v(\alpha)=0$ and $\varphi \in \mathbb{F}$, Lemma 2.3 gives

$$
D_{\alpha}(x)=E D_{\alpha}\left(x+Y_{\alpha}\right)-G_{\alpha}(x) .
$$

This is known as Poisson's equation for the random walk $S_{n}$. In order to prove our theorem, it is necessary to determine the behavior at $+\infty$ of the solution $D_{\alpha}(x)$ of this equation. The result we need would follow from the general theory of Poisson's equation if either the random walk were transient, or if it were recurrent and its distribution were either lattice or nonsingular (see Port and Stone (1969), for example). In our situation, however, these assumptions are not necessary for the desired conclusion, so we will give our own derivation of the necessary facts which takes advantage of special properties of our solution $D_{\alpha}(x)$. We begin with the case $v^{\prime}(\alpha)<0$. In this case $S_{n}$ is transient since

$$
E Y_{\alpha}=-\sum_{i=1}^{N} E W_{i}^{\alpha} \log W_{i}=-v^{\prime}(\alpha)>0 .
$$

Iterating (2.19) and passing to the limit, we see that

$$
D_{\alpha}(x)=\lim _{n \rightarrow \infty} E D_{\alpha}\left(x+S_{n}\right)-\sum_{k=0}^{\infty} E G_{\alpha}\left(x+S_{k}\right) .
$$

Here the sum is finite by Corollary 2.17, the renewal theorem (Theorem 1 of Sect. XI.9 of Feller (1966)), and the fact that $E Y_{\alpha}>0$, while the limit exists because $E D_{\alpha}\left(x+S_{n}\right)$ is increasing in $n$. Of course

$$
p(x)=\lim _{n \rightarrow \infty} E D_{\alpha}\left(x+S_{n}\right) \geqq D_{\alpha}(x)>0
$$

is harmonic for the random walk. Since $D_{\alpha}(x) e^{-\alpha x}$ is decreasing in $x$ and $D_{\alpha}(x) e^{(1-\alpha) x}$ is increasing in $x, p(x)$ is continuous. By the renewal theorem and Corollary 2.17,

$$
\sum_{k=0}^{\infty} E G_{\alpha}\left(x+S_{k}\right)
$$

is bounded on $R^{1}$ and tends to zero as $x \rightarrow+\infty$. Since $D_{\alpha}(x) \leqq e^{\alpha x}, \varlimsup_{x \rightarrow-\infty} p(x)<\infty$. Therefore since $E Y_{\alpha}>0, p(x)$ is constant in the nonlattice case and periodic of period $s$ in the lattice case. Hence

$$
\lim _{x \rightarrow \infty}[D(\alpha)-p(x)]=-\lim _{x \rightarrow \infty} \sum_{k=0}^{\infty} E G_{\alpha}\left(x+S_{k}\right)=0 .
$$

Putting $\theta=e^{-x}$ and recalling the definition of $D_{\alpha}$, it follows that

$$
\lim _{\theta \downarrow 0} \frac{1-\varphi(\theta)}{\theta^{\alpha} p(-\log \theta)}=1
$$

To check that in the lattice case 


$$
(-1)^{k} \frac{d^{k}}{d \theta^{k}}\left[\theta^{\alpha} p(-\log \theta)\right] \leqq 0
$$

for all $k \geqq 1$ and $\theta>0$, use the periodicity of $p$ to write

$$
\begin{aligned}
\theta^{\alpha} p(-\log \theta) & =\theta^{\alpha} p(-\log \theta+n s) \\
& =\theta^{n \alpha s}\left[\frac{\theta^{\alpha} e^{-n \alpha s} p(-\log \theta+n s)}{1-\varphi\left(\theta e^{-n s}\right)}\right]\left[1-\varphi\left(\theta e^{-n s}\right)\right] .
\end{aligned}
$$

Therefore

$$
\theta^{\alpha} p(-\log \theta)=\lim _{n \rightarrow \infty} e^{n \alpha s}\left[1-\varphi\left(\theta e^{-n s}\right)\right]
$$

Since $\varphi \in \mathbb{Q}$, it follows that the derivatives of $\theta^{\alpha} \rho(-\log \theta)$ have the correct signs. Note that since $\frac{1-\varphi(\theta)}{\theta}$ is monotone, if $\alpha=1$ then $p$ is both monotone and periodic, and hence constant. Turning now to the recurrent case, assume that $v^{\prime}(\alpha)=0$. Let $\tau$ be the first time that $S_{n}$ enters $(0, \infty)$, so that $S_{\tau}$ is the strict ascending ladder variable associated with $Y_{\alpha}$. Recall that $Y_{\alpha} \neq 0$ since $v \neq 0$ and that $E Y_{\alpha}=0$ since $v^{\prime}(\alpha)=0$. Therefore $\tau<\infty$ a.s. By (2.19),

$$
D_{\alpha}\left(x+S_{n}\right)-\sum_{k=0}^{n-1} G_{\alpha}\left(x+S_{k}\right)
$$

is a martingale. By the martingale stopping theorem,

$$
E D_{\alpha}\left(x+S_{\tau \wedge n}\right)-E \sum_{k=0}^{\tau \wedge n-1} G_{\alpha}\left(x+S_{k}\right)=D_{\alpha}(x) .
$$

By (2.15) and (3.6a) of Chap. XII of Feller (1966),

$$
E e^{\alpha S_{\tau}}<\infty
$$

Therefore, since $D_{\alpha}(x) \leqq e^{\alpha x}$ and $S_{n} \leqq S_{\tau}$ for $n<\tau$, we may pass to the limit in (2.21) to obtain

$$
E D_{\alpha}\left(x+S_{\tau}\right)-E \sum_{k=0}^{\tau-1} G\left(x+S_{k}\right)=D_{\alpha}(x) .
$$

This is again Poisson's equation, but for the random walk whose increments have the distribution of $S_{t}$. Put

$$
R(x)=E \sum_{k=0}^{\tau-1} G_{\alpha}\left(x+S_{k}\right)=E D_{\alpha}\left(x+S_{\tau}\right)-D_{\alpha}(x)
$$

By the duality lemma of Sect. XII.2 of Feller (1966),

$$
R(x)=\sum_{k=0}^{\infty} E G_{\alpha}\left(x+T_{k}\right)
$$

where $T_{k}$ is the random walk whose increments have the distribution of the weak descending ladder variable for the original random walk $S_{n}$. So, by the 
renewal theorem and Corollary 2.17 , there is a strictly positive continuous function $p(x)$ which is constant in the nonlattice case and periodic of period $s$ in the lattice case so that

$$
\lim _{x \rightarrow \infty}[R(x)-p(x)]=0 .
$$

Consider now the nonlattice case only, since the lattice case is handled similarly with derivatives and integrals being replaced by differences and sums respectively. Rewrite (2.23) as

$$
E \int_{0}^{S_{\tau}} D_{\alpha}^{\prime}(z+y) d y=R(z)
$$

Since $D_{\alpha}(x) \leqq e^{\alpha x},(2.14)$ and $(2.22)$ can be used to justify the interchange of expectation and integration in (2.25) to obtain

$$
\int_{0}^{\infty} D_{\alpha}^{\prime}(z+y) P\left(S_{\tau} \geqq y\right) d y=R(z) .
$$

Integrating both sides of this identity with respect to $z$ (using (2.14) and (2.22) again to justify the interchange of order of integration),

$$
\int_{0}^{\infty} D_{\alpha}(x+y) P\left(S_{\tau} \geqq y\right) d y=\int_{0}^{x} R(z) d z+c
$$

where $c=\int_{0}^{\infty} D_{\alpha}(y) P\left(S_{\tau} \geqq y\right)<\infty$. Since $D_{\alpha}(x) e^{-\alpha x}$ is decreasing in $x$,

$$
\frac{D_{\alpha}(x+y)}{D_{\alpha}(x)} \leqq e^{\alpha y}
$$

for $y \geqq 0$. Therefore, dividing (2.26) by $D_{\alpha}(x)$ and using (2.22), Theorem $2.12 \mathrm{~b}$, and the dominated convergence theorem, we see that

$$
\lim _{x \rightarrow \infty} \frac{\int_{0}^{\infty} R(z) d z+c}{D_{\alpha}(x)}=E S_{\tau},
$$

which is positive and finite. Therefore, by (2.24),

$$
\lim _{x \rightarrow \infty} \frac{D_{\alpha}(x)}{x}=\frac{1}{E S_{\tau}} \lim _{x \rightarrow \infty} \frac{1}{x} \int_{0}^{x} p(z) d z
$$

exists and is positive and finite. In the lattice case, the corresponding conclusion is that

$$
\lim _{n \rightarrow \infty} \frac{D_{\alpha}(x+n s)}{x+n s}=\frac{p(x)}{E S_{\tau}}
$$


for each $x \in R^{1}$. To deduce from this that

$$
\lim _{x \rightarrow \infty} \frac{D_{\alpha}(x)}{x p(x)}=\frac{1}{E S_{\tau}},
$$

proceed as follows. Let

$$
u_{n}(x)=\frac{D_{\alpha}(x+n s)}{x+n s}
$$

for $0 \leqq x \leqq s$. Then (2.27) asserts the pointwise convergence of $u_{n}$, while (2.28) asserts the uniform convergence of $u_{n}$ on $[0, s]$. But by the monotonicity of $D_{\alpha}(x) e^{-\alpha x}$ and (2.27), $D_{\alpha}(x) / x$ is bounded at $+\infty$. Hence by $(2.14), D_{\alpha}(x) / x$ has a uniformly bounded derivative at $+\infty$. Hence the family $\left\{u_{n}(x)\right\}$ is equicontinuous on $[0, s]$, so pointwise convergence implies uniform convergence. Part (b) of the theorem now follows from (2.28) with $p(x)$ replaced by $p(x) / E S_{\tau}$, by putting $\theta=e^{-x}$. The verification that $p(x) \in \mathfrak{P}_{\alpha, s}$ is the same as in the transient case, which was dealt with earlier in this proof.

\section{The Stable Transformation}

This section is devoted primarily to the construction of fixed points for $T$ of infinite mean. This is done by stopping a one sided stable process at a random time whose distribution is a fixed point of finite mean for a closely related smoothing transformation. This approach is natural since fixed points of finite mean have already been constructed under appropriate assumptions in Theorem 2.7, and since in view of Theorem 2.18, any fixed point must have stable like tails.

(3.1) Theorem. Suppose $v(\alpha)=0$ and $v^{\prime}(\alpha)<0$ for some $\alpha \in(0,1)$. Then $\mathfrak{F}$ is nonempty.

Proof. Define $\tilde{W}_{i}=W_{i}^{\alpha}$, and let $\tilde{v}, \tilde{T}$ and $\tilde{F}$ be defined in terms of $\left\{\tilde{W}_{1}, \ldots, \tilde{W}_{N}\right\}$ just as $v, T$ and $\mathfrak{F}$ were defined in terms of $\left\{W_{1}, \ldots, W_{N}\right\}$. Then $\tilde{v}(\beta)=v(\alpha \beta)$, so that $\tilde{v}(1)=v(\alpha)=0$ and $\tilde{v}^{\prime}(1)=\alpha v^{\prime}(\alpha)<0$. Therefore by Theorem 2.7, there is a $\psi \in \tilde{\widetilde{F}}$ with finite mean. Put

Then

$$
\varphi(\theta)=\psi\left(\theta^{\infty}\right)
$$

$$
\begin{aligned}
T \varphi(\theta) & =E \prod_{i=1}^{N} \varphi\left(\theta W_{i}\right) \\
& =E \prod_{i=1}^{N} \psi\left(\theta^{\alpha} W_{i}^{\alpha}\right) \\
& =\tilde{T} \psi\left(\theta^{\alpha}\right) \\
& =\psi\left(\theta^{\alpha}\right)=\varphi(\theta) .
\end{aligned}
$$

To check that $\varphi \in \mathfrak{F}$, it then suffices to verify that $\varphi$ is a Laplace transform. To see this, let $X(t)$ be the one sided stable process of index $\alpha$ whose Laplace 
transform is

$$
e^{-t \theta^{\alpha}}
$$

(see Sect. XIII.6 of Feller (1966), for example), and let $\tau$ be a random variable with Laplace transform $\psi$ which is independent of $X(\cdot)$. Then $X(\tau)$ has Laplace transform $\varphi$, since

$$
\begin{aligned}
& E\left(e^{-\theta X(\tau)}\right) E\left[E\left(e^{-\theta X(\tau)} \mid \tau\right)\right] \\
& =E\left[e^{-\theta^{\alpha} \tau}\right]=\psi\left(\theta^{\alpha}\right)=\varphi(\theta) .
\end{aligned}
$$

(3.2) Theorem. Suppose that $\mathfrak{F}$ is nonempty and that $v$ is not identically zero. Then

(a) $v(0)>0$, and

(b) for any $\varphi \in \mathfrak{F}, \varphi(\infty)$ is equal to the unique fixed point in $[0,1)$ of the function

$$
f(t)=\sum_{k=0}^{N} t^{k} P\left[\sum_{i=1}^{N} 1_{\left\{W_{i}>0\right\}}=k\right] .
$$

Proof. For $\varphi \in \mathbb{F}$,

$$
\varphi(\theta)=E \prod_{i=1}^{N} \varphi\left(\theta W_{i}\right)
$$

Therefore, taking the limit as $\theta \uparrow \infty$, we see that

$$
\begin{aligned}
\varphi(\infty) & =E \prod_{i=1}^{N}\left[1_{\left\{W_{l}=0\right\}}+\varphi(\infty) 1_{\left\{W_{i}>0\right\}}\right] \\
& =f[\varphi(\infty)]
\end{aligned}
$$

so $f$ has a fixed point in the interval $[0,1)$. Since $f$ is convex, $f(1)=1$, and

$$
\begin{aligned}
f^{\prime}(1) & =\sum_{k=0}^{N} k P\left[\sum_{i=1}^{N} 1_{\left\{W_{\imath}>0\right\}}=k\right] \\
& =\sum_{i=1}^{N} P\left(W_{i}>0\right)=e^{v(0)},
\end{aligned}
$$

it follows that $v(0)>0$ or that $f(t)=t$ for all $t$. In the latter case,

$$
\sum_{i=1}^{N} 1_{\left\{W_{i}>0\right\}}=1 \text { a.s. }
$$

so if we let $W=W_{1}+\ldots+W_{N},(3.3)$ becomes

$$
\varphi(\theta)=E \varphi(\theta W)
$$

or in terms of random variables, $X=X W$. Taking logarithms and iterating, we see that this can only happen if $W \equiv 1$, in which case $W_{i}=1$ on $\left\{W_{i}>0\right\}$. This implies that $v$ is identically zero, which is ruled out by the assumptions of the 
theorem. Thus $v(0)>0$, and $f$ has a unique fixed point in $[0,1)$, which proves both parts of the theorem.

(3.5) Theorem. Suppose that $v$ is not identically zero, but that $v(\alpha)=0$ and $v^{\prime}(\alpha)$ $=0$ for some $\alpha \in(0,1]$. Then $\mathfrak{F}$ is not empty.

Proof. Since $v$ is strictly convex under the given assumptions, $v(\beta)>0$ and $v^{\prime}(\beta)<0$ for all $\beta \in(0, \alpha)$. Define

$$
W_{i, \beta}=W_{i} e^{-v(\beta) / \beta}
$$

for $1 \leqq i \leqq N$ and $\beta \in(0, \alpha)$, and let $v_{\beta}$ and $T_{\beta}$ be the corresponding function and smoothing transformation. Then

$$
\begin{aligned}
v_{\beta}(\delta) & =\log \left[E \sum_{i=1}^{N} W_{i, \beta}^{\delta}\right] \\
& =\log \left[e^{-\delta v(\beta) / \beta} E \sum_{i=1}^{N} W_{i}^{\delta}\right] \\
& =v(\delta)-\frac{\delta v(\beta)}{\beta} .
\end{aligned}
$$

Therefore $v_{\beta}(\beta)=0$ and $v_{\beta}^{\prime}(\beta)=v^{\prime}(\beta)-\frac{v(\beta)}{\beta}<0$ for $\beta \in(0, \alpha)$. Therefore $T_{\beta}$ has a nontrivial fixed point $\psi_{\beta}$ by Theorem 3.1. Note that the function $f$ of Theorem 3.2 does not depend on $\beta$, so by that theorem, $\psi_{\beta}(\infty)=t_{0} \in[0,1)$ is independent of $\beta$. Since $\psi_{\beta}(c \theta)$ is a fixed point of $T_{\beta}$ whenever $\psi_{\beta}(\theta)$ is, we may choose the fixed point $\psi_{\beta}$ so that

$$
\psi_{\beta}(1)=\frac{t_{0}+1}{2}
$$

Let $X_{\beta}$ be a random variable with Laplace transform $\psi_{\beta}$, and choose a sequence $\beta_{n} \uparrow \alpha$ so that $X_{\beta_{n}}$ converges vaguely to $X \leqq \infty$ as $n \rightarrow \infty$. Put $\varphi(0)=1$ and

$$
\varphi(\theta)=E\left(e^{-\theta X}, X<\infty\right)
$$

for $\theta>0$. Then $\psi_{\beta_{n}}(\theta) \rightarrow \varphi(\theta)$ for all $\theta$, so in particular $\varphi(1)=\frac{1+t_{0}}{2}$. Since $\psi_{\beta}$ is a fixed point for $T_{\beta}$,

$$
\psi_{\beta}(\theta)=E \prod_{i=1}^{N} \psi_{\beta}\left(\theta W_{i} e^{-v(\beta) / \beta}\right)
$$

Since $v\left(\beta_{n}\right) \rightarrow 0$, we may pass to the limit as $n \rightarrow \infty$ to obtain

$$
\varphi(\theta)=E \prod_{i=1}^{N} \varphi\left(\theta W_{i}\right)
$$


Letting $\theta \downarrow 0$, we see that $\varphi(0+)=f[\varphi(0+)]$, where $f$ is the function defined in Theorem 3.2. Since

$$
\varphi(0+) \geqq \varphi(1)>t_{0},
$$

it follows that $\varphi(0+)=1$. Therefore $X<\infty$ a.s., so $\varphi \in \mathbb{Q}$ and hence $\varphi \in \mathbb{F}$ by (3.6) and the fact that $\varphi(1)<1$.

\section{The Associated Branching Random Walk}

This section is devoted to the proof of the convergence theorem for iterates of $T$. This convergence theorem also plays a role in the characterization of $\mathscr{F}$ given in Theorem 2a from the introduction. It is convenient to introduce the following discrete time branching random walk on $[-\infty, \infty)$. At each unit of time, each individual produces $N$ offspring and then dies. If $x$ is the position of the parent, then the offspring are placed at the positions $\left\{x+\log W_{1}, \ldots, x\right.$ $\left.+\log W_{N}\right\}$, where the vector $\left(W_{1}, \ldots, W_{N}\right)$ is chosen independently by each parent. Note that this is well defined even if $x$ or $\log W_{i}$ is $-\infty$. Let $\eta_{n}$ be the configuration of the branching random walk at time $n$. Since

$$
E^{\eta} \prod_{\eta_{1}(x) \geqq 1}\left[\varphi\left(e^{x}\right)\right]^{\eta_{1}(x)}=\prod_{\eta(x) \geqq 1}\left[T \varphi\left(e^{x}\right)\right]^{\eta(x)},
$$

a simple iteration yields

$$
T^{n} \varphi(\theta)=E^{\{\log \theta\}} \prod_{\eta_{n}(x) \geqq 1}\left[\varphi\left(e^{x}\right)\right]^{\eta_{n}(x)} .
$$

Let $L_{n}=\max \left\{x: \eta_{n}(x) \geqq 1\right\}$ be the position of the rightmost particle.

(4.1) Lemma. Suppose that $v$ is not identically zero, but that $v(\alpha)=0$ for some $\alpha>0$. Then

$$
\lim _{n \rightarrow \infty} L_{n}=-\infty \quad \text { a.s. }
$$

Proof. Since $v$ is not identically zero and $v(\alpha)=0$ for some $\alpha>0$,

$$
P\left(\max _{1 \leqq i \leqq N} W_{i}=1\right)<1 .
$$

Therefore, if $P\left(\max _{1 \leq i \leq N} W_{i} \leqq 1\right)=1, L_{n} \rightarrow-\infty$ a.s. trivially. On the other hand, if $P\left(\max _{1 \leqq i \leqq N} W_{i}>1\right)>0$,

$$
P\left(\limsup _{n \rightarrow \infty} L_{n}=-\infty\right)+P\left(\limsup _{n \rightarrow \infty} L_{n}=+\infty\right)=1 .
$$

Therefore it suffices to show that $\sup L_{n}<\infty$ a.s. In order to do this, let $\alpha>0$ be such that $v(\alpha)=0$. Then

$$
M_{n}=\sum_{x: \eta_{n}(x) \geqq 1} \eta_{n}(x) e^{\alpha x}
$$


is a (nonnegative) martingale, since

$$
E \sum_{i=1}^{N} e^{\alpha \log W_{i}}=E \sum_{i=1}^{N} W_{i}^{\alpha}=1
$$

This martingale was used by Kahane and Peyriere (1976) and by Kingman (1975). By the martingale convergence theorem, $\lim _{n \rightarrow \infty} M_{n}$ exists and is finite a.s. Since $e^{\alpha L_{n}} \leqq M_{n}$, it follows that $\sup L_{n}<\infty$ a.s., thus completing the proof of the lemma.

(4.2) Theorem. Assume that $v$ is not identically zero. Suppose that $\varphi \in \mathfrak{F}$ and $\psi \in$ ‥ If

then

$$
\lim _{\theta \downarrow 0} \frac{1-\varphi(\theta)}{1-\psi(\theta)}=1,
$$

$$
\lim _{n \rightarrow \infty} T^{n} \psi=\varphi
$$

Proof. By Theorems $3.2 \mathrm{a}$ and $2.12 \mathrm{a}, v(0)>0$ and $v(\alpha)=0$ for some $\alpha \in(0,1]$. Let $\alpha$ be the smallest such value. Then $v^{\prime}(\alpha) \leqq 0$. By Theorem 2.18 ,

and

$$
\liminf _{\theta \downarrow 0} \frac{1-\varphi(c \theta)}{1-\varphi(\theta)}=c^{\alpha} \min _{0 \leqq x \leqq s} \frac{p(-\log c+x)}{p(x)}
$$

$$
\limsup _{\theta \downarrow 0} \frac{1-\varphi(c \theta)}{1-\varphi(\theta)}=c^{\alpha} \max _{0 \leqq x \leqq s} \frac{p(-\log c+x)}{p(x)}
$$

for $c>0$, where $p \in \mathfrak{P}_{\alpha, s}$. Since $p \in \mathfrak{P}_{\alpha, s}, \theta^{\alpha} p(-\log \theta)$ is strictly increasing on $[0, \infty)$. Therefore

and

$$
\liminf _{\theta \downarrow 0} \frac{1-\varphi(c \theta)}{1-\varphi(\theta)}>1 \quad \text { if } c>1
$$

$$
\limsup _{\theta \downarrow 0} \frac{1-\varphi(c \theta)}{1-\varphi(\theta)}<1 \quad \text { if } c<1
$$

Fix $c>1$ and put

$$
\underline{\varphi}(\theta)=\varphi(c \theta) \text { and } \bar{\varphi}(\theta)=\varphi\left(c^{-1} \theta\right) .
$$

Then $\underline{\varphi}, \bar{\varphi} \in \mathfrak{F}$ and for some $\theta_{0}>0$ and all $0<\theta \leqq \theta_{0}$,

Therefore

$$
\underline{\varphi}(\theta) \leqq \psi(\theta) \leqq \bar{\varphi}(\theta) .
$$

$$
\prod_{\eta_{n}(x) \geqq 1}\left[\varphi\left(e^{x}\right)\right]^{\eta_{n}(x)} \leqq \prod_{\eta_{n}(x) \geqq 1}\left[\psi\left(e^{x}\right)\right]^{\eta_{n}(x)} \leqq \prod_{\eta_{n}(x) \geqq 1}\left[\bar{\varphi}\left(e^{x}\right)\right]^{\eta_{n}(x)}
$$


on the event $\left\{L_{n} \leqq \log \theta_{0}\right\}$. Since $\bar{\varphi}(\theta) \leqq 1$, Lemma 4.1 now gives

$$
\begin{aligned}
\liminf _{n \rightarrow \infty} T^{n} \varphi(\theta) & \leqq \liminf _{n \rightarrow \infty} T^{n} \psi(\theta) \\
& \leqq \limsup _{n \rightarrow \infty} T^{n} \psi(\theta) \\
& \leqq \limsup _{n \rightarrow \infty} T^{n} \bar{\varphi}(\theta) .
\end{aligned}
$$

Since $T^{n} \underline{\varphi}=\varphi$ and $T^{n} \bar{\varphi}=\bar{\varphi}$, it follows that all limit points of $T^{n} \psi(\theta)$ lie between $\overline{\varphi(c \theta)}$ and $\varphi\left(c^{-1} \theta\right)$. Since $c>1$ is arbitrary,

$$
\lim _{n \rightarrow \infty} T^{n} \psi(\theta)=\varphi(\theta)
$$

\section{The Totality of Fixed points; Moments}

From Theorems 2.7, 3.1 and 3.5, we now know that if $v(\alpha)=0$ and $v^{\prime}(\alpha) \leqq 0$ for some $\alpha \in(0,1]$, then $\mathfrak{F}$ is nonempty. Furthermore, Theorem 2.18 limits the possible behavior at the origin of any $\varphi \in \mathfrak{F}$. The first result in this section says that each behavior permitted by Theorem 2.18 in fact can occur.

(5.1) Theorem. Suppose that $v$ is not identically zero and that $v(\alpha)=0$ and $v^{\prime}(\alpha) \leqq 0$ for some $\alpha \in(0,1]$. If $p \in \mathfrak{P}_{\alpha, s}$, then there is a unique $\varphi \in \mathfrak{F}$ so that

and

$$
\lim _{\theta \downarrow 0} \frac{1-\varphi(\theta)}{\theta^{\alpha} p(-\log \theta)}=1 \quad \text { if } \quad v^{\prime}(\alpha)<0
$$

$$
\lim _{\theta \downarrow 0} \frac{1-\varphi(\theta)}{\theta^{\alpha} p(-\log \theta)|\log \theta|}=1 \quad \text { if } \quad v^{\prime}(\alpha)=0 .
$$

Proof. The uniqueness comes from Theorem 4.2. If the problem is nonlattice or $\alpha=1$, then $\mathfrak{B}_{\alpha, s}$ consists only of constants, so this result follows from Theorems $2.7,2.18,3.1$ and 3.5. So, we can assume that $\alpha<1$ and $s>0$. Let $g(\theta)=e^{-\theta}$ if $v^{\prime}(\alpha)<0$ and

$$
g(\theta)=\frac{2}{\pi} \int_{0}^{\infty} \frac{e^{-\theta x}}{1+x^{2}} d x
$$

which is asymptotic to $1-\theta|\log \theta|$ as $\theta \downarrow 0$, if $v^{\prime}(\alpha)=0$. Then by criterion 2 of Sect. XIII.4 of Feller (1966), since $p \in \mathfrak{P}_{\alpha, s}$ it follows that

$$
\psi(\theta)=g\left[\frac{\theta^{\alpha} p(-\log \theta)}{\alpha}\right]
$$

is in $\mathfrak{P}$. It is easy to check that

$$
\lim _{\theta \downarrow 0} \frac{1-\psi(\theta)}{\theta^{\alpha} p(-\log \theta)}=1 \quad \text { if } v^{\prime}(\alpha)<0
$$


and

$$
\lim _{\theta \downarrow 0} \frac{1-\psi(\theta)}{\theta^{\alpha} p(-\log \theta)|\log \theta|}=1 \quad \text { if } v^{\prime}(\alpha)=0
$$

By Theorems 3.1 and $3.5, \mathfrak{F}$ is nonempty. Take $\tilde{\psi} \in \mathfrak{F}$, which by Theorem 2.18 satisfies

and

$$
\lim _{\theta \downarrow 0} \frac{1-\tilde{\psi}(\theta)}{\theta^{\alpha} \tilde{p}(-\log \theta)}=1 \quad \text { if } v^{\prime}(\alpha)<0
$$

$$
\lim _{\theta \downarrow 0} \frac{1-\tilde{\psi}(\theta)}{\theta^{\alpha} \tilde{p}(-\log \theta)|\log \theta|}=1 \quad \text { if } v^{\prime}(\alpha)=0
$$

for some $\tilde{p} \in \mathfrak{P}_{\alpha, s}$. Since $\tilde{p} \in \mathfrak{P}_{\alpha, s}, \theta^{\alpha} \tilde{p}(-\log \theta)$ is strictly increasing on $[0, \infty)$ and is 0 at 0 and tends to $\infty$ at $\infty$. Hence $u(\theta)$ can be defined by

$$
[u(\theta)]^{\alpha} \tilde{p}[-\log u(\theta)]=\theta^{\alpha} p(-\log \theta) .
$$

By the periodicity of $p$ and $\tilde{p}, u$ satisfies

$$
u\left(\theta e^{s}\right)=u(\theta) e^{s}
$$

Therefore, if we define $\varphi(\theta)=\tilde{\psi}[u(\theta)]$, it follows that

and hence that

$$
\varphi\left(\theta W_{i}\right)=\tilde{\psi}\left[u\left(\theta W_{i}\right)\right]=\tilde{\psi}\left[u(\theta) W_{i}\right],
$$

$$
\begin{aligned}
E \prod_{i=1}^{N} \varphi\left(\theta W_{i}\right) & =E \prod_{i=1}^{N} \tilde{\psi}\left[u(\theta) W_{i}\right] \\
& =\tilde{\psi}[u(\theta)]=\varphi(\theta) .
\end{aligned}
$$

Furthermore, since $u(\theta) / \theta$ is bounded away from 0 and $\infty$ on $(0, \infty)$,

Hence

$$
\lim _{\theta \downarrow 0} \frac{\log u(\theta)}{\log \theta}=1
$$

and

$$
\lim _{\theta \downarrow 0} \frac{1-\varphi(\theta)}{\theta^{\alpha} p(-\log \theta)}=1 \quad \text { if } v^{\prime}(\alpha)<0
$$

$$
\lim _{\theta \downarrow 0} \frac{1-\varphi(\theta)}{\theta^{\alpha} p(-\log \theta)|\log \theta|}=1 \quad \text { if } v^{\prime}(\alpha)=0,
$$

by the corresponding properties of $\tilde{\psi}$. It remains to show that $\varphi \in \mathbf{Q}$. In order to do this, it suffices to note that $T^{n} \psi \in \mathfrak{Q}$ for all $n$ and to use the argument of the proof of Theorem 4.2 to show that

$$
\lim _{n \rightarrow \infty} T^{n} \psi=\varphi
$$

The point of the next result is to show that $\mathfrak{P}_{\alpha, s}$ is relatively large for $\alpha \in(0,1)$ and $s>0$. For simplicity, we will take $s=2 \pi$. 
(5.2) Theorem. Take $\alpha \in(0,1)$, let $a_{n}$ and $b_{n}$ be numbers which satisfy

and let

$$
\sum_{n=1}^{\infty} \sqrt{\left(a_{n}^{2}+b_{n}^{2}\right) \prod_{j=0}^{\infty}\left[1+\frac{n^{2}}{(j-\alpha)^{2}}\right]} \leqq 1
$$

Then $p \in \mathfrak{P}_{\alpha, 2 \pi}$.

$$
p(x)=1+\sum_{n=1}^{\infty}\left[a_{n} \sin n x+b_{n} \cos n x\right]
$$

Proof. Let $D$ denote differentiation. Then it is easy to check by induction that for $k \geqq 1$,

$$
D^{k}\left[\theta^{\alpha} p(-\log \theta)\right]=\theta^{\alpha-k}(-1)^{k}\left[\prod_{j=0}^{k-1}(D+j-\alpha) p\right](-\log \theta) .
$$

So, it suffices to show that

$$
\left.\prod_{j=0}^{k-1}(D+j-\alpha) p\right](x) \leqq 0
$$

for $k \geqq 1$ and $x \in R$. Define $a_{n}^{(k)}, b_{n}^{(k)}$ and $c^{(k)}$ by

$$
\left[\prod_{j=0}^{k-1}(D+j-\alpha)\right] p(x)=-c^{(k)}\left[1+\sum_{n=1}^{\infty}\left[a_{n}^{(k)} \sin n x+b_{n}^{(k)} \cos n x\right]\right] .
$$

Then $c^{(0)}=-1, a_{n}^{(0)}=a_{n}, b_{n}^{(0)}=b_{n}, c^{(k+1)}=(k-\alpha) c^{(k)}$,

$$
a_{n}^{(k+1)}=a_{n}^{(k)}-\frac{n}{k-\alpha} b_{n}^{(k)}
$$

and

$$
b_{n}^{(k+1)}=b_{n}^{(k)}+\frac{n}{k-\alpha} a_{n}^{(k)} .
$$

Therefore $c^{(k)} \geqq 0$ for $k \geqq 1$ and

$$
\left[a_{n}^{(k+1)}\right]^{2}+\left[b_{n}^{(k+1)}\right]^{2}=\left(1+\frac{n^{2}}{(k-\alpha)^{2}}\right)\left\{\left[a_{n}^{(k)}\right]^{2}+\left[b_{n}^{(k)}\right]^{2}\right\}
$$

so iterating,

$$
\left[a_{n}^{(k)}\right]^{2}+\left[b_{n}^{(k)}\right]^{2} \leqq\left(a_{n}^{2}+b_{n}^{2}\right) \prod_{j=0}^{\infty}\left[1+\frac{n^{2}}{(j-\alpha)^{2}}\right] .
$$

By the assumption, then,

$$
\sum_{n=1}^{\infty}\left\{\left[a_{n}^{(k)}\right]^{2}+\left[b_{n}^{(k)}\right]^{2}\right\}^{1 / 2} \leqq 1
$$

for $k \geqq 1$. The result then follows from the fact that

$$
\sup _{x}|a \sin x+b \cos x|=\sqrt{a^{2}+b^{2}} \text {. }
$$


The proof of the following theorem follows that of the corresponding result in Kahane and Peyriere (1976).

(5.3) Theorem. Suppose $v(1)=0$ and $v^{\prime}(1)<0$. Then for any $\mu \in \mathfrak{F}$ and $\beta>1$,

$$
\int_{0}^{\infty} x^{\beta} d \mu<\infty \quad \text { if and only if } v(\beta)<0 .
$$

Proof. Let $X, X_{1}, \ldots, X_{N}$ be independent random variables with distribution $\mu$. Suppose first that $E X^{\beta}<\infty$. Then write

$$
\begin{gathered}
X^{\beta} \stackrel{\mathcal{D}}{=}\left(\sum_{i=1}^{N} W_{i} X_{i}\right)^{\beta} \\
\geqq \sum_{i=1}^{N} W_{i}^{\beta} X_{i}^{\beta},
\end{gathered}
$$

where the inequality is strict on a set of positive probability. Then

$$
E X^{\beta}>E X^{\beta} \sum_{i=1}^{N} E W_{i}^{\beta}
$$

so that $v(\beta)<0$. For the converse suppose $v(\beta)<0$ and let $k$ be the integer which is determined by $k<\beta \leqq k+1$. Then for $x_{i} \geqq 0$,

$$
\begin{aligned}
\left(\sum_{i=1}^{N} x_{i}\right)^{\beta} & \leqq\left(\sum_{i=1}^{N} x_{i}^{\beta /(k+1)}\right)^{k+1} \\
& =\sum_{i=1}^{N} x_{i}^{\beta}+\sum c_{j_{1}, \ldots, j_{N}}\left(x_{1}^{j_{1}} \ldots x_{N}^{j_{N}}\right)^{\beta /(k+1)}
\end{aligned}
$$

for appropriate constants $c_{j_{1}, \ldots, j_{N}}$. Note that only terms with $\max _{i} j_{i} \leqq k$ and $\sum_{i=1}^{N} j_{i} \leqq k+1$ appear in the second summation. Therefore, if $Y, Y_{1}, \ldots, Y_{N}$ are independent and identically distributed nonnegative random variables, it follows that

$$
E\left(\sum_{i=1}^{N} Y_{i} W_{i}\right)^{\beta} \leqq\left(E Y^{\beta}\right) e^{v(\beta)}+c\left[E Y^{k}\right]^{\beta / k}
$$

for some $c$ which may depend on $\beta$ but not on the distribution of $Y$. This can be restated as

$$
\int_{0}^{\infty} x^{\beta} d(T v) \leqq e^{v(\beta)} \int_{0}^{\infty} x^{\beta} d v+c\left[\int_{0}^{\infty} x^{k} d v\right]^{\beta / k}
$$

for any $v \in \mathfrak{M}$. Recalling from the proof of Theorem 2.7 that

$$
\mu=\lim _{n \rightarrow \infty} T^{n} \delta_{1}
$$


where $\delta_{1}$ is the pointmass at 1 , we see that $v(\beta)<0$ and $\int_{0}^{\infty} x^{k} d \mu<\infty$ imply that $\int_{0}^{\infty} x^{\beta} d \mu<\infty$. Since $v(\beta)<0$ and $v(1)=0$ imply that $v<0$ on $(1, \beta]$, the previous statement can be iterated to complete the proof of the theorem. In the first step of the iteration, of course, $\int_{0}^{\infty} x d \mu<\infty$ is used, but that is part of the statement of Theorem 2.7.

\section{References}

1. Dawson, D.A.: The critical measure diffusion process. Z. Wahrscheinlichkeitstheorie verw. Gebiete 40, 125-145 (1977)

2. Durrett, R. An infinite particle system with additive interactions. Advances in Appl. Probability 11, 355-383 (1979)

3. Feller, W.: An introduction to probability theory and its applications, Vol. II. New York: J. Wiley and Sons 1966

4. Harris, T.E.: A correlation inequality for Markov processes in partially ordered state spaces. Ann. Probability 5, 451-454 (1977)

5. Holley, R., Liggett, T.M.: Generalized potlatch and smoothing processes. Z. Wahrscheinlichkeitstheorie verw. Gebiete 55, 165-195 (1981)

6. Kahane, J.P., Peyriere, J.: Sur certaines martingales de Benoit Mandelbrot. Advances in Math. 22, 131-145 (1976)

7. Kallenberg, O.: Stability of critical cluster fields. Math. Nachr. 77, 7-43 (1977)

8. Kerstan, J., Matthes, K., Mecke, J.: Infinitely divisible point processes. New York: J. Wiley and Sons 1978

9. Kingman, J.F.C.: The first birth problem for an age-dependent branching process. Ann. Probability 3, 790-801 (1975)

10. Liggett, T.M.: Random invariant measures for Markov chains, and independent particle systems. Z. Wahrscheinlichkeitstheorie verw. Gebiete 45, 297-313 (1978)

11. Liggett, T.M., Spitzer, F.: Ergodic theorems for coupled random walks and other systems with locally interacting components. Z. Wahrscheinlichkeitstheorie verw. Gebiete 56, 443-468 (1981)

12. Port, S.C., Stone, C.J.: Potential theory of random walky on abelian groups. Acta Math. 122, 19-114 (1969)

13. Royden, H.: Real Analysis, second edition. New York: MacMillan 1968

14. Spitzer, F.: Infinite systems with locally interacting components. Ann. Probability 9, 349-364 (1981)

15. Stone, C.J.: On a theorem of Dobrushin. Ann. Math. Statist. 39, 1391-1401 (1968)

Received February 1983

Added in Proof. After completion of this paper, B. Mandelbrot suggested that some of the remarks and conjectures in his papers in C.R. Acad. Sci. Paris, Series A (volume 278, pp. 289-292 and 355358) might be directly relevant to our work. The closest point of contact appears to be his conjecture in paragraph 17 , which our results show is incorrect. 